www. revis tad y o. com

\title{
Esquema de condicionantes en la relación proveedor-cliente en la indus- tria automotriz. Caso sector autopartes en la Zona del Bajío ${ }^{1}$
}

Outline of conditions in the supplier-customer relationship in the automotive industry. The auto parts sector in the Bajío

\author{
Pedro Aguilar-Pérez y Lucila Patricia Cruz-Covarrubias \\ Centro Universitario de Ciencias Económico Administrativas (CUCEA), de la Universidad de Guadalajara (Mexico) \\ paguilar_17@hotmail.com; patriciacruz2204@hotmail.com
}

Fecha de recepción: 13-09-2014

Fecha de aceptación: 10-04-2015

Resumen: El objetivo del trabajo es analizar las condiciones que favorecen las relaciones proveedor-cliente en empresas automotrices en la región Bajío, en un contexto de productividad y cadena de suministro. Se establece cómo las armadoras condicionan el proceso productivo y suministro de proveedores de primer nivel (Tier1). La investigación aborda un método cualitativo se sustenta en la técnica de entrevista semiestructurada en cinco empresas(Tier1) de una muestra de 38 proveedores automotrices. Las conclusiones indican, que las relaciones entre armadoras y proveedores(Tier1) tienen más que un sustento comercial de compra-venta, son más complejas ya que incorporan innovación; procesos de producción; calidad; costos, entre otros. Como resultado es que hay una clara relación corporativa derivada del país de origen, lo cual se expresa en: desarrollo de productos, decisiones de nuevas inversiones y los términos de las negociaciones de proveeduría.

Palabras clave: Industria automotriz, relaciónes proveedor-cliente, sector autopartes, proveedor Tier1.

Abstract (English) The main objective of this research paper is to analyze the conditions/relations that take hold between the vendor (suppliers) and its clients (customer), regarding of the supply chain and productivity within the automotive industry located in the region of Bajío. The original equipment manufacturers (OEM) condition the productivity process and the purveying of its major suppliers of parts known as a Tier 1 company. The methodology used in this study will be qualitative, premising on the semi-structured interview technique administer to five Tier 1 companies, from a sample of 38 . The underlying findings reveal that the relation between OEMs and Tier 1 corporations are much deeper than a simple purchasing-sale interaction. In fact it involves a great degree of innovations, productions processes, quality, costs etc... All of this resulting in a clear corporative relationship derived from the country of origin, which is expressed in; product development, new investment decisions and terms of negotiations of purveyors.

Keywords: automotive industry, relations vendor-client, suppliers of parts Tier 1.

\section{Introducción}

En la actualidad el sector de autopartes es parte de la cadena de valor automotriz y provee dos mercados: el de equipo original, que se usa directamente en la fabricación de automóviles nuevos, y el mercado de repuestos (chasis, interiores, exteriores, eléctricos y electrónicos). En el 2012, el sector automotriz y autopartes representaron un $20.8 \%$ del total de la inversión extranjera directa (IED) en México.
La industria de autopartes se ha incrementado en el mercado con gran eficacia, por las alianzas que han surgido con las armadoras, por citar algunos ejemplos, Ford hizo alianza con Grupo Alfa para dar apertura a Nemak, ésta se dedica a la fabricación de cabezas para motor de aluminio. General Motors y Grupo Condumex también se aliaron para la proveeduría de arneses. Ford con Grupo Vitro y Grupo Visa crearon Vitroflex, siendo este el productor de vidrio automotriz y Carplastic es el fabricante de tableros de plástico. Estas estrategias corporativas benefician a empresas

\footnotetext{
${ }^{1}$ Este artículo es producto del Proyecto de Investigación que se encuentra realizando el Cuerpo Académico UDG-CA-669 de la institución Universidad de Guadalajara que inicio en 2013 sobre la "Articulación Productiva del Sector Automotriz en la Región Centro-Occidente de México, Implicaciones Económicas y Sociales para Impulsar el crecimiento”.
} 
mexicanas, sea grande, mediana y pequeña ya que desarrollan alta tecnología en sus procesos de producción, cumpliendo con los estándares de calidad para exportar y con una alta reducción de costos de producción.

Indudablemente, en este contexto industrial globalizado, una de las estrategias más comunes de las armadoras es la de producir modelos idénticos en varios mercados, lo que les permite fabricar automóviles en diferentes países con los mismos componentes. De allí que, al menos en el caso de los elementos más críticos o estratégicos, las armadoras procuren que el abastecimiento de sus plantas productivas -cualquiera que sea su ubicación geográfica $\square$ provenga del mismo proveedor. Para ello alienta o presionan a los proveedores para que se expandan geográficamente, de modo que establezcan, sea mediante inversiones conjuntas, adquisición de empresas o radicación directa de nuevas filiales, plantas productivas que se ubiquen cerca de las armadoras.

El tema sobre las relaciones que establecen las empresas no es nuevo, ha sido tratado ampliamente por varios estudiosos desde distintas perspectivas. Sin embargo, es a partir de la década de los sesenta, ante el éxito alcanzado por las empresas japonesas, que las relaciones de proveeduría empiezan a ser eje central de las estrategias empresariales como también de cuestiones académicas. Carrillo y González López (1999) mencionan que "el llamado modelo productivo "fordista" es cuestionado de raíz por el sistema de producción japonés y la producción flexible en pequeños lotes sustentada en un conjunto de empresas, llegando a constituirse los atributos de estos en referentes de la modernización y competitividad empresarial" (1999, p. 94).

Al respecto, Sarache, Castrillon y Ortiz (2009) referenciando a (Kamman y Bakker, 2004) señalan que la tendencia actual que se debe considerar en la relación con los proveedores es que estos forman parte integral de la cadena de abastecimiento; en consonancia con este planteamiento; (mencionando a Vijay, 2006 y Chung, Sternquist y Chen, 2008) señalan que una empresa no compite sola, pues forma parte de una red dentro de la cual debe competir en forma colaborativa, con la finalidad de mejorar la calidad, entrega y productividad, mientras que simultáneamente reduce sus costos. Tal planteamiento se debe a una tendencia mundial que establece pasar de la competencia entre firmas a la competencia entre cadenas productivas. En este sentido, trazan que la construcción y la gestión de relaciones cliente-proveedor re reconocen actualmente como uno de los pilares fundamentales para la creación de ventaja competitiva sostenible.

Sarache, Castrillon y Ortiz (2009), en concordancia con Araz y Ozkarahan (2007) y Choy, Lee y Lau (2005), plantean que las relaciones cliente-proveedor pueden ser de dos tipos: "de simple intercambio comercial y de socios estratégicos" (p. 151). El primer tipo se refiere a mantener una buena relación comercial, donde esta no se piensa en un proceso a largo plazo, ni se ve al proveedor como fuente de ventaja competitiva. En el segundo tipo, la finalidad primordial es convertir a los proveedores en aliados estratégicos a partir de sólidas relaciones comerciales colaborativas. Señalan los autores que esta tendencia "viene imponiéndose desde hace más de 15 años. De hecho, el outsourcing, como tendencia mundial en la gestión logística, se fundamenta en esquemas de este tipo" (2009, p. 151).

Con base en estos postulados se realizó la presente investigación, que tiene relevancia científica en tanto aporta nuevos conocimientos en esta rama. Vista desde el panorama de la economía mexicana y regional en su conjunto, la industria automotriz es liderara por consorcios transnacionales, está orientada hacia la exportación de bienes, representa una dinámica muy acentuada de cambios en sus productos, tecnología, en su organización de trabajo y relaciones laborales, así como un importante aportación al PIB.

Además, este sector ofrece una multitud de configuraciones de relaciones comerciales, laborales e industriales diferentes que esta diversidad no se encuentra en ningún otro sector manufacturero de la economía mexicana.

Considerando el aspecto académico, la investigación aporta elementos administrativos, comerciales y productivos que llevan a cabo las empresas automotoras con sus clientes (armadoras), con el fin de que alumnos, académicos e interesados en el tema, tengan un panorama general de las relaciones productivas en una industria y región especifica, lo que permitirá a estos contar con un conocimiento actual, claro y pertinente del sector productivo.

La contribución de este estudio es de tipo empírico en lo referente a la relación cliente-proveedor de empresas del sector automotriz, desde las perspectivas relacionadas con los modos de producción más competitivos. Al ser un estudio cientifico se hace relevante investigar las variables que impulsan las relaciones cliente-pro- 
veedor y de gran interes su grado de impacto.

De este modo, al situarse en un contexto de relación proveedor-cliente, donde el papel del cliente es representado por empresas armadoras, y el del proveedor por empresas del sector de autopartes, el objetivo de este trabajo es analizar los factores que condicionan las relaciones cliente-proveedor en empresas automotrices de primer nivel Tier ${ }^{2}{ }^{2}$ en la zona del Bajío, en un contexto de competencia globalizada y reestructuración productiva. Dos fueron los cuestionamientos centrales que guiaron el trabajo: ¿cómo se conforman las relaciones de proveeduría de las empresas?, y ¿cuáles son las principales características de estas relaciones?

Este documento se estructura en cinco apartados. Después de la introducción, la primera parte trata sobre la revisión conceptual de algunos de los principales planteamientos sobre las relaciones cliente-proveedor entre empresas del sector automotriz. El segundo apartado, presenta un panorama actual del sector automotriz en la Zona del Bajío, específicamente en la ciudad de San Luis Potosí.

En la tercera parte se exponen la metodologia utilizada en el estudio. En la cuarta parte se presentan los principales resultados de la investigación de campo que se realiz a empresas proveedoras automotrices en la región del Bajío en San Luis Potosí, con el objeto de identificar las condicionantes en las relaciones cliente-proveedor entre ellas, como el potencial que representa esta región para mayores inversiones extranjeras. Finalmente, en la última parte se sintetizan los resultados obtenidas, donde se evidenció cuales son los factores que determinan las relaciones de proveeduría que se basan en experiencias de larga duración basadas en formar parte de grandes corporativos con capacidades productivas, financieras y tecnológicas.

\section{La relación proveedor-cliente en el sector automotor}

Carrillo y González López, mencionan que para hablar de relaciones cliente-proveedor, probablemente se tenga en primera instancia una memoria a los "planteamientos neoclásicos de Walras y Marshall, sobre su aspecto puramente económico-comercial, donde predominan acuerdos entre empresas sobre precio, calidad y tiempo de entrega, principalmente. Asimismo, la proximidad física entre las empresas involucradas constituye una ventaja de localización porque significa disminución de los costos de transportación y de los tiempos de entrega" (1999, p. 94).

Carrillo y González (1999), señalan que también es necesario incorporar otras condicionantes más de orden tecnológico, organizativo y cultural, estrechamente relacionadas con el origen del capital de las empresas, las cuales son de particular importancia ante las nuevas reglas de la competencia y ante las formas de reestructuración productiva y territorial.

Por otro lado, el enfoque interactivo, propuestos por Hákansson (1982) y conocido como Industrial Marketing and Purchasing (IMP), basado en la observación de la relación entre clientes (grandes grupos empresariales) y sus proveedores en ambientes industriales, ha concluido que los factores más importantes que afectan la relación proveedor-cliente son la tecnología acumulada, la estructura y el tamaño de la empresa.

Carrizo Moreira (2007), señala que de acuerdo con Ring y Van de Ven (1992), múltiples compañías comenzaron a comprometerse en relaciones inter-organizacionales debido al aparecimiento de tecnologías emergentes, a la presión competitiva y a las decisiones estratégicas. Moreira menciona, que es evidente que en mercados altamente competitivos, como el sector automotriz, las formas relacionales citadas se usen con frecuencia para acceder a nuevos mercados y para ganar economías de escala. Moreria, indica "es imposible mantener relaciones intensas con un elevado número de proveedores, por lo que las estrategias cooperativas entran en juego (2007, p. 95).

Según Carrizo Moreira $(2007$, p. 95) referenciando a Laming (1993) y a Dyer (1996), mencionan que los principales beneficios de una relación proveedor-cliente son los siguientes:

- Un proveedor puede fácilmente acceder a la tecnología del socio.

- $\quad$ Se generan ventajas competitivas a largo plazo, por ejemplo, mayores cuotas de mercado, mayor velocidad en el desarrollo cooperativo de nuevos productos (DCNP), mejorar en la calidad, menores costos, etc.

- Se generan mejoras en la productividad de cor-

\footnotetext{
${ }^{2}$ Clasificación internacional de las empresas proveedoras del sector automotriz que implica diferentes niveles de proveeduría, Tier1 es proveedor de equipo original básico, motores, transmisión, aire acondicionado, entre otros más, para las plantas armadoras; Tier2 entrega algunos complementos de equipo a Tier1, por ejemplo: uniones, tornillos, complementos metal mecánicos.
} 
to plazo, menores inventarios y mejor control de calidad.

- $\quad$ Originan menores costos en investigación y desarrollo, producción y distribución.

\begin{abstract}
- $\quad$ Permiten menores riesgos financieros en inversiones conjuntas.
\end{abstract}

- $\quad$ Fomentan el compromiso a largo plazo.

La relación proveedor-cliente, ha sido un tema fundamental que forma parte de los principios que sustentan la cadena de suministro moderna. Jiménez, cita que "desde hace tiempo existe una gran cantidad estudios y autores (Hendrick y Ellram, 1993; Lambert y Gardner, 1996; Giménez, 2000; Rey, 2001; Holweg, et al, 2005) que han desarrollado ensayos sobresalientes de esta temática: la mayoría de ellos basándose en las relaciones cliente-proveedor de la industria del automóvil" (2006, p. 31).

El asunto discutido con mayor amplitud en este sector es el cambio de las relaciones de confrontación basado en el precio, a un modelo de relaciones de colaboración. De hecho, Martínez y Pérez (2004), reconocen que las relaciones que los ensambladores tienen con sus proveedores influyen en el precio y en la calidad de los componentes de un automóvil.

Los criterios de precio y calidad de los productos son factores importantes que las empresas ensambladoras tienen en consideración para elegir a sus proveedores de primer nivel. Sin embargo, actualmente las compañías más avanzadas establecen que un proveedor, también debe contar con procesos y productos de alta calidad y tecnología; crear un enfoque total hacia los clientes en términos de servicio; localizar las plantas cerca de sus instalaciones; y convertirse en una empresa innovadora y creativa, ampliando las actividades de investigación y desarrollo.

A partir de estas condiciones, en el sector automotriz las relaciones ensambladoras-proveedor se han tornado mucho más complejas que en otros sectores. Martínez y Pérez (2004), señalan que ese tipo de relaciones han experimentado un cambio importante desde mediados de los años ochenta, como consecuencia de la reestructuración de este sector y de la difusión global de un conjunto de prácticas de gestión. Reportan que antiguamente predominaban las renovaciones anuales de contratos, un número elevado de proveedores por componente, y una competencia entre ellos basada casi exclusivamente en el precio; actualmente los es- tándares de esta industria evidencian que los contratos se extienden como mínimo durante la vida de un modelo; que se ha reducido el número de proveedores por componente; y que la competencia se basa fundamentalmente en: a) calidad del producto; b) ingeniería; c) plazo y puntualidad en la entrega; d) mejor precio; e) mayor facilidad para adaptarse a la empresa ensambladora; y f) mayores niveles de integración tecnológica.

Por su parte, Lamming (1993), señala que el modelo de suministro ajustado en el sector automotriz puede caracterizarse bajo los siguientes nueve factores principales: 1) naturaleza de la competencia; 2) base para la toma de decisiones de aprovisionamiento; 3) intercambio de información; 4) la gestión de la capacidad; 5) prácticas de entrega; 6) negociación de cambio de precio; 7) actitud hacia la calidad; 8) investigación y el desarrollo; y 9) nivel de presión en la relación; todos estos surgidos de las relaciones comerciales entre ensambladores y proveedores.

En el sector del automóvil, con el grado de desintegración vertical que posee, las restricciones de proximidad que pueden plantearse en las relaciones cliente-proveedor, van a tener una particular importancia en la explicación de la problemática espacial para el abasto, sobre todo entre los ensambladores de automóviles y los proveedores de primer nivel (Tier 1).

En definitiva, las relaciones entre empresas de autopartes y sus clientes deben estar sustentadas por sistemas avanzados que permita integrar sus procesos de gestión y producción.

\section{Sector automotriz en San Luis Potosí}

La Secretaria de Desarrollo Económico (SDE), menciona que en 2012 el universo de la industria automotriz y de autopartes en el Estado de San Luis Potosí era de 103 unidades: una armadora y 102 proveedoras. Por su número de trabajadores, el $5 \%$ del total son microempresas, $19 \%$ pequeñas, $41 \%$ medianas y $35 \%$ grandes empresas (SDE, 2012).

La S.D.E., de San Luis Potosí, establece que cuatro características destacan en las compañías instaladas en el Estado: 1) el capital de 48 empresas fue cien por ciento extranjero; 2) existen 38 empresas certificadas bajo la norma ISO/TS; 3 ) el $52.9 \%$ de los insumos que utilizaron las empresas en la generación de sus productos es de origen nacional y; 4) se instaló en el 
Estado (Municipio de Villa de Reyes) la empresa de ensamble automotriz: General Motors de México, S. de R.L. de C.V. (Complejo San Luis Potosí).

Las 38 empresas proveedoras Tier 1 han logrado desarrollar un mayor grado de automatización y flexibilidad productiva, simplificando sus procesos de producción, reduciendo los costos de ensamblaje e incrementando la sistematización de sus procesos, permitiéndoles adaptarse a las necesidades de las grandes armadoras. Gran parte de estas empresas cuentan con altos niveles de calidad y tienen importantes alianzas tecnológicas y centros para el desarrollo e innovación tecnológica, proveen de componentes a las armadoras que se encuentran en el país, así como las que se localizan en el extranjero principalmente en Estados Unidos de América.

Por su parte, las 64 empresas proveedoras Tier2 y Tier3 están integradas principalmente por capital nacional y local. Las empresas Tier2 realizan actividades de proveeduría de soporte a las Tier1 como piezas maquinadas, fundiciones, estampados y forjas, entre otras. En el caso de las empresas Tier3, estas llevan a cabo actividades de proveeduría de soporte a las Tier2 como acabados, procesos de corte, doblez, formado y pintura entre otras.

La ubicación geográfica de San Luis Potosí en el centro de la República permite a su planta automotriz y de autopartes tener acceso rápido a la mayoría de las empresas ensambladoras del país. El hecho de que el Estado se encuentre equidistante de las ciudades de México, Monterrey y Guadalajara, y que esté comunicado por carretera y ferrocarril a los más importantes puntos del territorio nacional, entre otros, y a los puertos de Tampico y Altamira en el Golfo de México; Lázaro Cárdenas y Manzanillo en el Océano Pacífico, así como a las ciudades fronterizas de Brownsville, Mc Allen y Laredo, TX., por las que se realiza un alto porcentaje de comercio exterior, está contribuyendo a que sea una de las más diversificadas economías de México y una región que incrementa sus intercambios de inversión, comercio y cultura, y amplíe su participación fundamentalmente con los países de América del Norte, Europa y Latinoamérica.

Su comercio exterior se facilita a través de la instalación de Puntos de Despacho Aduanero, y de la Aduana y el Recinto Fiscalizado en el Aeropuerto Internacional de San Luis Potosí.

\section{Metodología}

El método de investigación se aborda desde un enfoque cualitativo a partir de una primera fase de revisión documental para analizar el concepto de relación proveedor-cliente y como los proveedores de autopartes desarrollan dicha relación con las ensambladoras de automóviles, a través de artículos en revistas científicas, libros, tesis, informes, y su relación con los implicados en el tema que nos compete. En la segunda fase se realizó investigación de campo en la que se recolectó información empírica a través de la entrevista semiestructurada, que como lo indica la técnica, la parte libre permite profundizar en las características específicas de los entrevistados. Por ello, admite una mayor libertad y flexibilidad en la obtención de información (Hernández-Sampieri: 2010). Se contactó en esta instancia de la investigación a cinco gerentes de empresas de autopartes (Tier1) establecidas en la ciudad de San Luis Potosí. La investigación se llevó a cabo en los meses de enero a abril de 2014.

La entrevista fue aplicada mediante un muestreo por conveniencia, donde las empresas (proveedores automotrices) se eligieron de acuerdo a su fácil disponibilidad (Mejía: 2014). Se buscó en todo momento que las organizaciones tuvieran una relación comercial con la armadora General Motors (GM). El análisis y procesamiento de la información se realizó a través de una transcripción manual (desgravada de entrevistas) y ordenada en esquemas en función de las preguntas que guiaron la entrevista.

La entrevista consta de dos apartados: 1) información general de la organización y 2) de la relación con empresas del sector automotriz y su vinculación con otras instituciones sociales. Las preguntas versaban sobre el conocimiento de por qué deciden instalarse en la región potosina, su origen de capital, tamaño de la empresa y sus productos que manufactura, así como también, información sobre sus clientes, proveedores, su vinculación con los organismos gubernamentales y educativos, y asociaciones empresariales, también para que expresaran, su punto de vista con respecto a sus expectativas en el mercado del sector automotriz, y recomendaciones para generar un encadenamiento productivo en el estado. 


\section{Descripción de los casos investigados}

Las principales características de los casos analizados en esta investigación, se realizan desde el punto de vista descriptivo y estratégico de la empresa. Las empresas proveedoras automotrices analizadas son de tamaño grande Tier 1, esto determinado tanto por su volumen de ventas como por el número de empleados. Se puede afirmar que el tamaño es un factor fundamental, dado que las mayores empresas están tecnológicamente mejor capacitadas que las pequeñas.

\section{1. (Proveedor Lear Corporation) Caso E1}

La E1es una empresa grande de capital de origen estadounidense, cuenta con 560 trabajadores, con un gran volumen de ventas en el mercado nacional como en el extranjero. Produce asientos para el automóvil AVEO, y la vestidura de la camioneta TRAX ambos automóviles de la armadora GM, así como también corredores, costuras y mecanismos de seguros de asiento. Provee a la empresa INSA, esqueletos para asientos de NISSAN y Honda.

La E1 tienen varios clientes en el extranjero, por lo regular empresas grandes y medianas en el sector automotriz. Cuenta con una estructura organizacional bien fundamentada para el logro de sus objetivos. Tecnológicamente, la compañía está concentrada en el desarrollo de competencias productivas y de calidad. La empresa cuenta con la certificación de la Norma ISO/ TS $16949^{3}$

La relación comercial que tiene con su cliente GM es a través de contrato que se realiza en la ciudad de Michigan en Estados Unidos, la duración de este contrato es de 5 o 6 años, los pedidos se hacen a través de sistema digital. La empresa recibe las especificaciones de sus clientes y trabajan en conjunto para el desarrollo de nuevos productos. La Planta automotriz realiza evaluaciones anuales al proveedor, para asegurar el cumplimiento de las especificaciones del producto, la seguridad de entrega, en tiempo y forma. El personal de la empresa está capacitado y entrenado para ser multidivisional, para cumplir con los requerimientos de la armadora GM. En resumen los requisitos fundamen- tales para asegurar la relación comercial con la Planta automotriz son: capacidad de respuesta productiva; Investigación y Desarrollo I\&D; Justo a Tiempo, Costo y Calidad.

\section{2. (Proveedor San Luis Rassini) Caso E2}

La E2 es una empresa multinacional, grande con 123,000 empleados directos en todo el mundo, y un volumen de ventas de 39 billones de dólares. Produce y diseña discos, tambores, mazas y ensambles-bujes hule-metal, para el mercado nacional y norteamericano de vehículos ligeros y pesados. Tiene dos plantas en México, una establecida en Coahuila y la otra en el Estado de México. Cuenta con una cartera de clientes de armadoras entre las que incluye a Ford, General Motors, Honda, Nissan, Toyota, Maserati, TRW, entre otras. Es una empresa proveedora en el nivel Tier1, pero también dentro de la cadena productiva del sector automotriz en Tier2.

Cuenta con las certificaciones de calidad de ISO/TS 16949, industria limpia e ISO-1400. Ha desarrollado una capacidad tecnológica adecuada, lo que le permite la satisfacción del cliente. Sus competencias tecnológicas y logísticas le han permitido posesionarse como líder en el mercado internacional en suspensión y sus partes.

La relación con sus clientes es a través de un contrato que establecen ambas compañías, que es a más de tres años. El tipo de producto, cantidad y calidad la condiciona los clientes (armadoras). Las terminales de autos señalan las características y especificaciones del producto (muelle, barra de torsión, resorte y/o disco), a través de una cinta de cartilla, así le llaman en su relación comercial. La compañía diseña el producto y la tecnología establecida y el cliente únicamente avala el diseño y producto.

Las armadoras continuamente <<cada año>> realizan evaluaciones a la empresa, donde cumpla con las especificaciones establecidas en el contrato, sobre todo en competitividad de producción y calidad de productos; con certificaciones y premios de calidad en los procesos de producción y logística. La tecnología de punta es fundamental para la producción de los pro-

\footnotetext{
${ }^{3}$ La norma ISO/TS 16949 es el desarrollo de un sistema de gestión de calidad con el objetivo de una mejora continua enfatizando en la prevención de error y en la reducción de desechos de la fase de producción. TS 16949 se aplica en las fases de diseño/desarrollo de un nuevo producto, producción y, cuando sea relevante, instalación y servicio de productos relacionados con el mundo de la automoción. Está basado en el estándar ISO 9000. Los requisitos son aplicables a lo largo de toda la cadena de producción. En la actualidad también las plantas armadoras de vehículos se están confrontando con esta norma del ISO.
} 
ductos y eso lo avala la armadora (cliente), así como también implantar el sistema Justo a tiempo e inversión en investigación y desarrollo I\&D. La experiencia en el sector de autopartes cuenta mucho para la empresa y para la relación comercial que con los clientes.

\section{3. (Proveedor DENSO) Caso E3}

La empresa E3, empresa grande que a nivel mundial depende de DENSO Corporation que es una división de Toyota en el ramo electrónico, es la segunda empresa de autopartes a nivel mundial. Inicio operaciones en México desde 1994 con dos plantas, tiene presencia en 38 países, con más de 140,000 empleados y con ventas de 39.08 mdd anuales. Los productos en los que participa en el mercado nacional y extranjero, son: cuadros de instrumentos, panel de control de temperatura, transmisor de combustible, módulo de aire y combustible, sensor de velocidad de la rueda, comprobación de fugas evaporador, válvulas de control de aceite y sensor de posición del acelerador.

Para la empresa el proceso de producción es parte fundamental para la satisfacción de sus clientes, por lo que la compañía hace la mayoría de su propio equipo, lo que permite adaptar e incorporar ideas innovadoras para impulsar continuamente la calidad y la eficiencia. DENSO se basa en avanzados procesos de producción, en desarrollo de tecnología, en robótica de alta precisión, en sistemas de inspección de visión artificial y de métodos precisos para llevar a cabo pruebas de calidad y controles durante el proceso de producción. La empresa cumple y va más allá de los requisitos de la industria y las expectativas del cliente para las pruebas de calidad. Tienen certificaciones de calidad internacional y nacional, así como gran cantidad de premios de empresas, organismos y asociaciones, en General Motors, por ejemplo, le han otorgado por más de diez años, el Premio de "proveedor del año".

La relación con sus clientes, como Toyota, Honda, GM, Ford, Chrysler, VW y Nissan, entre otros, es a través de licitaciones de productos y no realizan contratos con estas armadoras. Para poder tener derecho a licitar con la armadora, primeramente se tiene que estar certificado con normas de calidad, desarrollar alta tecnología y capacidad de producción y entrega, una vez que se cumple con estos requerimientos, se participa en la licitación y posteriormente en la asignación. Hay una evaluación constante por parte de los clientes, ya que la relación es por licitación de producto, este se encuentra en constante auditoria y evaluación. Se resume que la relación comercial con los clientes es a través de añadirle valor continuo al proceso de producción y por consiguiente al producto final.

\section{4. (Proveedor Autotek México) E4}

E4 es una empresa transnacional que nace en Octubre de 1991 en la Ciudad de Puebla México, con la finalidad de hacer una empresa de negocios de alta tecnología, calidad y eficiencia en los procesos de: Soldadura, Estampados y Pintura Automotriz. Es una unidad de negocios de la división de Cosma con sede en Alemania. Cuenta con 983 empleados en la planta. Manufactura y provee partes estampadas, ensambles y aplicación de pintura catafórica a la industria Automotriz de México, USA, Europa y Asia.

La empresa cuenta con la infraestructura, tecnología de punta y procesos de manufactura de los productos, por ello es que garantiza la satisfacción y calidad que ofrece en sus productos y servicios. Se ha esforzado continuamente por desarrollar nuevas tecnologías y métodos de producción con los más altos estándares de calidad, con el menor impacto en el medio ambiente. Los clientes (armadoras) solicitan de la compañía y de nuestros productos, que tengamos varias certificaciones, por lo que tenemos el ISO TS16949:2009, ISO 14001, Industria Limpia, “A” Quality Grade BY VWM, Proveedor del año.

La empresa es considerada Tier2, aunque en ocasiones es Tier1 ya que provee sus productos y servicios a empresas como: Ford; VW; NISSAN; Chrysler; GM y Lear. Ofrece productos y soporte técnico en lo referente al cromado, niquelado y cobrizo. La relación comercial con sus clientes es a través de concursos, dependiendo del producto y el cliente es como se hace la relación. En armadoras la mayoría de veces la relación es a través de concursos, en cambio con clientes proveedores Tier1 es a través de contratos. La relación con sus clientes se basa en una elevada calidad de sus productos, capacidad de producción, Justo a tiempo y costos. El incremento de su capacidad tecnológica en actividades de valor añadido, como la de concepción y de ingeniería de producto, le permitió a la E4 general una relación proveedor-cliente basada en el comprometimiento mutuo.

\section{5. (Proveedor Grupo ONEX) E5}

La E5 es parte del grupo Industrial y Automotriz de Tomkins Ltd., mismo que es propiedad de un consorcio formado por la canadiense Onex y Pension Plan Investment Board. Con sede en Denver, Colorado EE.UU., es líder mundial en ingeniería industrial y de fabricación. La compañía emplea a más de 14.500 empleados y tiene operaciones de ventas y de producción en todos los principales mercados del mundo, incluyendo América del Norte y del Sur, Europa, Asia, Australia y Oriente Medio. 
A pesar de ser pionera entre las industrias del Valle de Toluca, en la actualidad el equipo y tecnología de Mezclado de Hule, se encuentran entre los más avanzados en el ámbito mundial. En las Células de Manufactura, se fabrican productos de inigualable calidad que cumplen las normatividades y requerimientos de los clientes más exigentes (como son los de Equipo Original Automotriz), tanto en líneas nacionales como de exportación. La búsqueda de la excelencia en las operaciones de la empresa la ha llevado a incursionar en el desarrollo de procesos mediante la aplicación efectiva de la Manufactura Esbelta y las metodologías de Seis Sigma, dando así la principal característica de la Planta Toluca : "Ser una Operación de Manufactura de Clase Mundial". La Planta cuenta con importantes certificaciones y reconocimientos de calidad como son los siguientes:

\section{- $\quad$ Certificación ISO14001}

- $\quad$ Proveedor del año de GM en cinco diferentes ocasiones.

- Reconocimiento a la Excelencia Empresarial.

- Reconocimiento de Industria Limpia del Gobierno Federal

\section{- Reconocimiento de capacitación de CONALEP \\ - Reconocimiento por ahorro de agua por parte del Gobierno del Estado de México.}

Productos que se elaboran en la actualidad son: Banda en $V$ para Equipo Original y Reemplazo automotriz, Banda V, para servicio pesado industrial, Banda V, para servicio ligero industrial, Banda de Tiempo, Banda Transportadora, Banda de Transmisión, Manguera envuelta para manejo de materiales y uso petrolero, Manguera Trenzada Horizontal, Manguera Envuelta de Gran Diámetro, Manguera Curva Automotriz.

El tipo de producto y el tipo de cliente han condicionado la relación entre la E5 y sus clientes: como la empresa produce productos de plástico, normalmente tiene que seguir las especificaciones impuestas por sus clientes y las propuestas de nuevos productos. Así, aunque la E5 esté comprometida con satisfacer las exigencias de las terminales, a fin de fortalecer las relaciones, ha encontrado dificultades, debido a que las decisiones son tomadas en niveles muy altos de la compañía, aunque la empresa aquí en México se consigue involucrarse en la creación de nuevos productos.

Tabla 1. Esquema de los casos estudiados

\begin{tabular}{|c|c|c|c|c|c|}
\hline & E1 & E2 & E3 & E4 & E5 \\
\hline $\begin{array}{l}\text { Empleados } \\
\text { directos }\end{array}$ & $\begin{array}{l}560 \text { empleados en } \\
\text { México. }\end{array}$ & $\begin{array}{l}\text { 4,700 colabora- } \\
\text { dores en USA, } \\
\text { México y Brasil. }\end{array}$ & $\begin{array}{l}140,000 \text { empleados } \\
\text { en todo el mundo. }\end{array}$ & $\begin{array}{l}983 \text { empleados en } \\
\text { México. }\end{array}$ & $\begin{array}{l}14,500 \text { empleados } \\
\text { en todo el mundo }\end{array}$ \\
\hline Tipo de productos & $\begin{array}{l}\text { Asientos para Autos } \\
\text { completos; Vestidu } \\
\text { as para asiento; Es- } \\
\text { queleto de Asiento. }\end{array}$ & $\begin{array}{l}\text { Barras de Torsión/ } \\
\text { resortes heli- } \\
\text { coidales/Bujes de } \\
\text { hule y metal. }\end{array}$ & $\begin{array}{l}\text { Diferentes Pan- } \\
\text { eles de control y } \\
\text { sensores de aire, } \\
\text { gasolina, velocidad, } \\
\text { etc. }\end{array}$ & $\begin{array}{l}\text { Partes estampadas, } \\
\text { ensambles y apli- } \\
\text { cación de pintura } \\
\text { catoforética. }\end{array}$ & $\begin{array}{l}\text { Diferentes tipos de } \\
\text { bandas y mangeras. }\end{array}$ \\
\hline Normas de calidad & ISO/TS16949 & $\begin{array}{l}\text { ISO/TS } 16949, \\
\text { industria limpia e } \\
\text { ISO-1400 }\end{array}$ & $\begin{array}{l}\text { No menciona certi- } \\
\text { ficación especifica, } \\
\text { pero si cuenta con } \\
\text { ellas. }\end{array}$ & $\begin{array}{l}\text { ISO/TS16949; ISO } \\
\text { 14001, Industria } \\
\text { Limpia, "A" Quality } \\
\text { Grade BY VWM }\end{array}$ & $\begin{array}{l}\text { Certificación ISO } \\
14001\end{array}$ \\
\hline Perfil Internacional & $\begin{array}{l}\text { Proveedor Tier1. } \\
\text { Capital de origen } \\
\text { estadounidense. } \\
\text { Exportación del es- } \\
\text { queleto del asiento. }\end{array}$ & $\begin{array}{l}\text { Exporta a USA y } \\
\text { provee a difer- } \\
\text { entes armadoras. } \\
\text { Proveedor Tier1. }\end{array}$ & $\begin{array}{l}\text { Exporta al extranje- } \\
\text { ro y tiene presencia } \\
\text { en } 38 \text { paises. }\end{array}$ & $\begin{array}{l}\text { Exporta y servicio a } \\
\text { armadoras en USA, } \\
\text { Europa y Asia. }\end{array}$ & $\begin{array}{l}\text { Operaciones de } \\
\text { ventas y de pro- } \\
\text { ducción en todos } \\
\text { los principales mer- } \\
\text { cados del mundo, } \\
\text { incluyendo América } \\
\text { del Norte y del } \\
\text { Sur, Europa, Asia, } \\
\text { Australia y Oriente } \\
\text { Medio. }\end{array}$ \\
\hline
\end{tabular}




\begin{tabular}{|c|c|c|c|c|c|}
\hline $\begin{array}{l}\text { Influencia del } \\
\text { cliente en la selec- } \\
\text { ción del proveedor }\end{array}$ & $\begin{array}{l}\text { Proveedor Tier1. } \\
\text { Capital de origen } \\
\text { estadounidense. }\end{array}$ & $\begin{array}{l}\text { Proveedor Tier1 y } \\
\text { 2. Plantas en USA } \\
\text { y Brasil. Centro de } \\
\text { Desarrollo tecno- } \\
\text { logico en Plymouth, } \\
\text { Michigan. Alianza } \\
\text { con NHK de Japón, } \\
\text { lider en tecnología } \\
\text { para suspensiones. }\end{array}$ & $\begin{array}{l}\text { Proveedor Tier1. } \\
\text { Los productos son } \\
\text { de Japón y Estados } \\
\text { Unidos. Empresa } \\
\text { de origen de capital } \\
\text { Japón. Exporta a } \\
\text { Europa, America } \\
\text { y Asia. Se instala } \\
\text { donde estan sus } \\
\text { clientes. }\end{array}$ & $\begin{array}{l}\text { Proveedor Tier1 y } \\
\text { 2. Es unidad de ne- } \\
\text { gocios de la división } \\
\text { Cosma. Exporta a } \\
\text { USA, Europa y Asia. } \\
\text { Pertenece al grupo } \\
\text { Magna. }\end{array}$ & $\begin{array}{l}\text { Es parte del grupo } \\
\text { Industrial y Auto- } \\
\text { motriz de Tomkins } \\
\text { Ltd., mismo que es } \\
\text { propiedad de un } \\
\text { consorcio formado } \\
\text { por la canadiense } \\
\text { Onex y Pension } \\
\text { Plan Investment } \\
\text { Board. Con sede en } \\
\text { Denver, Colorado } \\
\text { EE.UU., es líder } \\
\text { mundial en ingeni- } \\
\text { ería industrial y de } \\
\text { fabricación. }\end{array}$ \\
\hline $\begin{array}{l}\text { Desarrollo del } \\
\text { Producto }\end{array}$ & $\begin{array}{l}\text { Cada vez se } \\
\text { está innovando } \\
\text { cumpliendo con } \\
\text { los estándares } \\
\text { establecidos con los } \\
\text { clientes. }\end{array}$ & $\begin{array}{l}\text { Se busca la } \\
\text { innovación y la tec- } \\
\text { nología en la propia } \\
\text { empresa. }\end{array}$ & $\begin{array}{l}\text { La innovación en } \\
\text { los Centros de } \\
\text { Investigación en } \\
\text { Estados Unidos y } \\
\text { Europa. }\end{array}$ & $\begin{array}{l}\text { La empresa desar- } \\
\text { rolla su tecnologia } \\
\text { y sistemas de } \\
\text { producción, esto } \\
\text { para satisfacer a } \\
\text { sus clientes. }\end{array}$ & $\begin{array}{l}\text { Desarrolla su propia } \\
\text { tecnología y de la } \\
\text { más avanzada en el } \\
\text { mundo. Su sistema } \\
\text { de manufactura con } \\
\text { los más altos estan- } \\
\text { dares de calidad. }\end{array}$ \\
\hline $\begin{array}{l}\text { Factores que forta- } \\
\text { lecen la relación }\end{array}$ & $\begin{array}{l}\text { El establecimiento } \\
\text { de contratos por lar- } \\
\text { go plazo; Exclusiv- } \\
\text { idad de productos. } \\
\text { Empresa multina- } \\
\text { cional. Adecuada a } \\
\text { las exigencias de la } \\
\text { industria. Produc- } \\
\text { tos de calidad y } \\
\text { logistica. }\end{array}$ & $\begin{array}{l}\text { Contratos a largo } \\
\text { plazo y las certifica- } \\
\text { ciones de calidad; } \\
\text { Productos con las } \\
\text { especificaciones } \\
\text { establecidas e } \\
\text { inversión en I\&D. } \\
\text { Suministro de } \\
\text { productos a tiempo, } \\
\text { calidad y cantidad. }\end{array}$ & $\begin{array}{l}\text { La calidad del pro- } \\
\text { ducto que ofrecen; } \\
\text { Empresa Europea; } \\
\text { cumplimiento en } \\
\text { las licitaciones; la } \\
\text { implantación de } \\
\text { sistemas de calidad, } \\
\text { y el desarrollo e } \\
\text { inversión en su tec- } \\
\text { nología y procesos. }\end{array}$ & $\begin{array}{l}\text { Concursos y con- } \\
\text { tratos a mediano } \\
\text { y largo plazo; el } \\
\text { cumplimiento de } \\
\text { estandares de cali- } \\
\text { dad; empresa multi- } \\
\text { nacional; la calidad } \\
\text { de sus productos. }\end{array}$ & $\begin{array}{l}\text { Empresa multina- } \\
\text { cional; contratos } \\
\text { establecidos en el } \\
\text { corporativo; se cum- } \\
\text { ple con las especifi- } \\
\text { caciones del cliente; } \\
\text { el cumplimiento de } \\
\text { estandares de cali- } \\
\text { dad. Empresa lider } \\
\text { en el sector. }\end{array}$ \\
\hline
\end{tabular}

\section{Conclusiones}

La participación del sector automotriz en el desarrollo económico de México ha sido constante desde principios del siglo $\mathrm{XX}$ y sigue marcando la pauta en la segunda década del siglo XXI, la posición estratégica de nuestro país en el contexto internacional gracias a los recursos naturales con los que cuenta, a la capacidad y bondad de la mano de obra y a condiciones de logística y operaciones en algunas zonas de la república favorece la fabricación de vehículos ligeros y pesados por lo que la ubicación de las plantas ha detonado la derrama económica para la mejora de las condiciones de la población.

Los resultados de la investigación nos señalan claramente que para lograr una estabilidad de las relaciones proveedor-cliente hay varios patrones según la complejidad tecnológica de los componentes sumi- nistrados. Las relaciones de las armadoras con sus proveedores tiende a ser de mediano a largo plazo. Una vez que se le asigna la entrega de un sistema y/o componente a un proveedor, la relación permanece mientras se produzca el modelo de auto o autos correspondientes, siempre que se cumplan las estipulaciones de calidad, entrega, producción y costos. Esto sin duda disminuye los costos de transacción y favorece las inversiones que los fabricantes de autopartes deben realizar para adecuar su capacidad productiva a las demandas de las armadoras.

Se puede interpretar, que las negociaciones proveedor-cliente se realizan a nivel corporativo, teniendo un mayor margen de negociación y pueden licitar sus productos.. Con respecto a los clientes nacionales las negociaciones las realizan predominantemente las propias plantas proveedoras. En todos los casos, el respaldo de capital y tecnológico del corporativo es 
fundamental. Las cinco empresas proveedoras no tienen exclusividad con una ensambladora. Salvo la E1, que siendo Tier1, provee exclusivamente el producto terminado (asiento) a una sola armadora (GM). Pero siendo Tier2 no cuenta con esa exclusividad. Las demás empresas proveedoras, destinan sus plantas actuales para la proveeduría con diferentes ensambladoras en México y para el mercado extranjero.

En resumen los proveedores de la industria automotriz tienen una relación con sus clientes basada en estrategias de calidad, de justo a tiempo, de producción y logística usando sistemas de información, donde existe una política activa de crear y desarrollar nuevos productos para enfrentar los aspectos técnicos de la evolución del mercado y tener como resultado la competitividad y una relación con el cliente de largo plazo. Calidad, productividad y capacidad de aprendizaje, son la fórmula para el éxito en mercados altamente competidos, como es el de la industria automotriz.

Se concluye que la confianza y la cooperación entre proveedor Tier1 y ensambladora es una característica común y esto parece ser la tendencia general en la industria del automóvil en la zona del Bajío; se ha incrementados la comunicación en los dos sentidos, el cliente colabora en la mejora de los proveedores, y la relación contractual suele plantearse -al menos inicialmente- a largo plazo. Aunque es importante señalar, que la mayor parte de los proveedores identifican el precio y calidad como variables fundamentales en su relación con el ensamblador, y se sienten presionados por su mayor poder de negociación.

A modo de conclusión, podemos marcar que la localización lejana o cercana entre proveedor y ensambladora no afecta las practicas comerciales, de involucramiento y aprovisionamiento; sin embargo, si influye la lejanía en las practicas denominadas operativas. Las entregas multidiarias en sistema Kanban no se pueden realizar de manera directa entre proveedores lejanos y las armadoras. La solución para mantener estas prácticas comerciales es la utilización en proximidad de centros logísticos que garanticen este flujo frecuente. El término es que el alejamiento del proveedor supondrá un mayor costo para cumplir los requisitos de entrega ajustada. Por tanto se puede mencionar que existe una tendencia hacia la búsqueda d proximidad con las ensambladoras establecidas en México.

Por último, las perspectivas de crecimiento de los proveedores de nivel uno Tier1, son muy positivas. El desarrollo de redes de proveeduría automotriz en México se ha fortalecido con los tratados comerciales, tanto en el mercado interno como en el extranjero y el TLCAN se ha fortalecido en forma sorprendente. Aún queda incierta la posibilidad de desarrollo de proveedores nacionales de segunda y tercera línea, así como por comprobarse la posibilidad de alcanzarse las proyecciones del gobierno sobre el desarrollo de empresas nacionales en un futuro cercano.

\section{Bibliografía}

AMIA (2010). La industria Automotriz mexicana frente a la situación económica actual: una agenda para el mercado interno. Boletín mensual de prensa, Presidencia de AMIA. Presentación a la Cámara de Diputados. Recuperado de: http://www.amia.com.mx

CARRILLO, J., y GONZÁLEZ, S. L. (1999). "Relaciones cliente-proveedor de empresas automotrices alemanas en México". Actes du GERPISA, 25, pp. 93-104. Recuperado de: http://gerpisa.org/ancien-gerpisa/actes/25/25-6.pdf

CARRIZO MOREIRA, A. (2007). "La internacionalización de Pymes industriales a través de Multinacionales. Presentación de Algunos casos de los sectores automotor y electrónico". Cuadernos de Administración, 20(34), pp. 89-114. Recuperado de: http:// www.scielo.org.co/pdf/cadm/v20n34/v20n34a05.pdf

HAKANSSON, H. (1982). "International marketing and purchasing of industrial goods: An interaction Approach". Suecia: University of Uppsala. Recuperado de: http://www.impgroup.org/uploads/books/ InternationalMarketing.pdf

HERNÁNDEZ-SAMPIERI, R., FERNÁNDEZ, C. y BAPTISTA, P. (2010). Metodología de la investigación ( $7^{\mathrm{a}}$ ed.). México, Editorial Mc Graw-Hill.

INA, INDUSTRIA NACIONAL DE AUTOPARTES (2012). Recuperado en: http://www.ina.com.mx

JIMÉNEZ, SÁNCHEZ, J. E. (2006). "Un análisis del sector automotriz y su modelo de gestión en el suministro de las autopartes". Publicación Técnica No 288, Secretaria de Comunicaciones y Transportes, Instituto Mexicano del Transporte.

LAMMING, R. (1993). Beyond Partnership Strategies for Innovation and Lean Supply. (Vol. 94). London: Prentice Hall. 
MARTíNEZ, S. Á. y PÉREZ P. M. (2004). "Evolución de la relación fabricante-proveedor del automóvil: modelos teóricos y evidencia empírica". Departamento de Economía y Dirección de Empresas. Universidad de Zaragoza. Seminario: Nueva configuración de la empresa industrial: Implicaciones para el sector de componentes del automóvil. Fundación Eduardo Barreiros y la Universidad Complutense de Madrid.
SARACHE CASTRO, W. A., CASTRILLÓN GÓMEZ, Ó. D. y ORTIZ FRANCO, L. F. (2009). "Selección de proveedores: una aproximación al estado del arte". Cuadernos de Administración, 22(38), pp. 145-168. Retrieved March 16, 2015. Recuperado de from http://www.scielo.org.co/scielo.php?script=sci_arttext\&pid=S0120-35922009000100008\&lng=en\&tIn$g=e s$. 